\title{
EchoGéo
}

25 | 2013

Moyen-Orient : espaces et passeurs du changement

\section{Le galérien, le révolté et le yuppie : trois trajectoires d'immigrés égyptiens à Abu Dhabi}

\section{Lucile Gruntz}

\section{(2) OpenEdition}

\section{Journals}

Édition électronique

URL : https://journals.openedition.org/echogeo/13522

DOI : $10.4000 /$ echogeo. 13522

ISSN : 1963-1197

\section{Éditeur}

Pôle de recherche pour l'organisation et la diffusion de l'information géographique (CNRS UMR 8586)

Référence électronique

Lucile Gruntz, « Le galérien, le révolté et le yuppie : trois trajectoires d'immigrés égyptiens à Abu Dhabi », EchoGéo [En ligne], 25 | 2013, mis en ligne le 10 octobre 2013, consulté le 11 août 2021. URL : http:// journals.openedition.org/echogeo/13522 ; DOI : https://doi.org/10.4000/echogeo.13522

Ce document a été généré automatiquement le 11 août 2021.

EchoGéo est mis à disposition selon les termes de la licence Creative Commons Attribution - Pas d'Utilisation Commerciale - Pas de Modification 4.0 International (CC BY-NC-ND) 


\title{
Le galérien, le révolté et le yuppie : trois trajectoires d'immigrés égyptiens à Abu Dhabi
}

\author{
Lucile Gruntz
}

\begin{abstract}
«Bon, maintenant, le père de Rim est d'accord pour que je l'épouse, même si je n'ai pas trouvé de travail ici d'ici début janvier, et qu'on n'a pas le sou... Il ne pouvait pas le dire avant, non? Je ne serais pas venu! Je le savais que les visas de long séjour et les permis de résidence sont très difficiles à obtenir pour les Égyptiens, mon frère et ma tante me l'avaient dit... Mais qu'est-ce que je pouvais faire, dismoi ? Et c'est encore pire depuis la révolution!». Adam, 28 ans, en quête d'un emploi de comptable (discussion, Abu Dhabi, décembre 2012).
\end{abstract}

1 Par-delà les ruptures politiques à répétition, la société égyptienne est marquée par des phénomènes à la pérennité remarquable. Initiée de concert avec la libéralisation de l'économie en 1971, l'émigration à destination des monarchies rentières du Golfe arabique est de ceux-ci. Les Émirats arabes unis figurent aujourd'hui parmi les destinations privilégiées des Égyptiens dans la péninsule : ils seraient environ 650000 à y résider ${ }^{1}$. Quant à leurs remises financières, elles représenteraient près de $16 \%$ du montant total de la rente migratoire perçue par l'Égypte, estimée de 8 à 9 millions de dollars américains annuels ${ }^{2}$. L'émigration vers les Émirats arabes unis revêt par conséquent une importance socioéconomique à la fois individuelle et collective en Égypte. Elle alimente un imaginaire doré de la fédération émirienne, mise en scène dans les récits des émigrés de retour et dans les médias égyptiens comme la terre des mille et une opportunités et des mille et un cabarets: le "rêve émirien » attire aventuriers en quête de fortune, d'emploi et de plaisirs, avant comme depuis la révolution entamée en janvier 2011. Et ce, malgré les innombrables obstacles administratifs, politiques et économiques qui balisent l'épopée migratoire, dont rend compte Adam, cité en exergue.

2 En dépit de son poids symbolique et économique en Égypte, l'immigration égyptienne dans le Golfe est mal servie par la littérature scientifique. Les travaux qualitatifs traitant de ses dimensions sociopolitiques demeurent rares. Les enquêtes ciblent plutôt 
l'émigration vers l'Europe (Müller-Mahn, 2005; Saad, 2007) ou les retours dans les villages du Delta du Nil (Weyland, 1993 ; Van Aken, 2005). Dans la péninsule arabique, d'autres communautés immigrées sont sous les feux de la rampe scientifique, les travailleurs originaires du sous-continent indien en particulier (Vora, 2013; Gardner, 2010). L'article tente de compenser ce vide à partir d'une double enquête ethnographique: l'une menée au Caire de 2006 à 2011 auprès de quarante-cinq ménages d'émigrés de retour des monarchies golfiennes, dont six des Émirats arabes unis ; l'autre initiée à Abu Dhabi et Dubaï en décembre 2012, auprès de vingt immigrés de nationalité égyptienne, dont dix-sept hommes. Il interroge les changements sociaux imputés et imputables à l'immigration égyptienne aux Émirats arabes unis.

3 Comment les immigrés content-ils leurs expériences émiriennes, leurs conséquences biographiques et leurs éventuelles répercussions collectives ? Comment les vécus différenciés de la condition immigrée interagissent-ils avec les inégalités et conflictualités prévalant dans les sociétés de départ comme d'arrivée?

\section{Eléments de méthode : la « pensée par cas » appliquée aux Émirats}

4 Les sociabilités professionnelles, familiales, amicales, amoureuses d'Adam, Nasir et $\mathrm{Galal}^{3}$, trois hommes égyptiens immigrés à Abu Dhabi, sont au cœur de cette contribution. Les récits et observations recueillis sur les deux terrains auprès d'autres migrants égyptiens, ainsi qu'aux Émirats arabes unis auprès d'immigrés américains, français, italiens, jordaniens, iraniens, palestiniens, bangladais, indiens, pakistanais, d'âges, genres et professions variés, ont permis de situer ces trois trajectoires en regard de la société égyptienne et de ses luttes, d'une part, et d'autre part, au sein du monde social d'Abu Dhabi, des liens qui s'y développent, des conflits qui y adviennent. Les trois cas sont éclairants par les recoupements auxquels ils appellent, par les nuances qu'ils rendent sensibles (Passeron et Revel, 2005) ; entre Égypte et Émirats arabes, ils esquissent un portrait contrasté des structures de la migration et de leur articulation aux trajectoires personnelles.

5 Ces dernières sont analysées à l'aune des rapports de pouvoir prévalant sur les lieux de la migration, ainsi que des interstices pratiques qu'y ménagent les immigrés. Par exemple, l'administration émirienne discrimine entre immigrés en fonction de leurs origines nationales et "aires culturelles» d'appartenance supposée (Kapiszewski, 2006). Engager une enquête à partir d'une «communauté » nationale donnée permet d'envisager l'efficacité sociale des catégorisations administratives, tout comme d'observer la subtilité de leurs actualisations quotidiennes. Les trajectoires d'Adam, Nasir et Galal illustrent le caractère consubstantiel et situé (Dorlin, 2009) des rapports de pouvoir à Abu Dhabi. Les imbrications entre règles administratives, rapports de classes, attentes familiales ou amoureuses, impératifs professionnels, amitiés communautaires ou cosmopolites, imprègnent les vies et les fortunes diverses des trois hommes.

6 Chaque trajectoire singulière nous informe sur les limites du « changement » associé à l'expérience migratoire : celui-ci varie selon que l'on soit chômeur ou banquier, fils de mécanicien ou de bourgeois, célibataire ou amoureux, polyglotte ou arabophone, émigré adolescent ou à l'âge adulte, arrivé dans les années 1970 ou les années 2000... 
Ces variations obéissent à des régularités sociologiquement analysables et à des effets de contextes : c'est là l'objet des lignes qui suivent.

\section{Dresser le décor : des politiques et des hommes}

7 Si Adam a le privilège de l'exergue, c'est que sa réflexion introduit au cœur des rapports de pouvoir qui favorisent les départs. L'urgence migratoire s'est accrue au gré de quarante ans de politiques d'austérité en Égypte, soldées notamment par un sousemploi et un chômage massif des nouveaux entrants sur le marché du travail, diplômés ou non. Le processus révolutionnaire n'a guère changé la donne migratoire : l'inflation a accru l'insécurité économique, sans que nulle mesure probante en faveur de l'emploi et des droits des travailleurs n'ait été mise en œuvre par les successeurs de l'ère Moubarak.

8 Assumer le coût de la vie et du mariage auquel il est difficile de faire face en Égypte, même en travaillant : l'extrait de citation présenté ci-dessus indique que le rêve émirien est d'autant plus entraînant que les rêveurs sont jeunes et pauvres. Il montre aussi que la réussite à laquelle aspirent les candidats au départ ne s'évalue pas uniquement en termes de gain matériel, mais encore de genre et de rétribution symbolique. La réussite financière permet d'accumuler la dot nécessaire au mariage, sésame vers la sexualité licite; elle conforte la prodigalité masculine, qui ouvre la voie à la reconnaissance des cadets par leurs aînés et fonde les inégalités domestiques. L'émigration doit permettre aux jeunes hommes de s'imposer socialement: de devenir des hommes sans l'épithète.

\section{Les immigrés en travailleurs}

9 Les rêves de réussite résistent plus ou moins bien à la confrontation aux politiques migratoires. Aux Émirats arabes unis, les immigrés sont avant tout des travailleurs, désignés comme tels (al-'umâl al-wâfidûn) dans les documents officiels, les médias et les interactions. Alors que les citoyens sont partiellement divertis du marché du travail par la redistribution de la rente orchestrée par les familles régnantes, le recours aux travailleurs étrangers de diverses nationalités et qualifications assure la main-d'œuvre nécessaire à l'économie des États fédérés, en dépit de politiques fédérales toujours plus restrictives (Lori, 2010). Comme le relèvent tous les enquêtés, les Égyptiens sont l'une des nationalités les plus représentées dans toutes les catégories professionnelles.

Les immigrés sont d'autant plus réduits à leur condition laborieuse que le séjour est conditionné par l'obtention d'un contrat de travail. Le système légal de la kafâla oblige chaque immigré à se placer, contre rétribution, sous le "parrainage » d'un employeur de nationalité émirienne. Ce parrainage peut être exercé directement par le patron dans les petites et moyennes entreprises ou indirectement dans les grandes entreprises ou le secteur public. Les variantes de la kafâla informent des parcours migratoires divers. Les cadres employés à des postes qualifiés dans les grandes entreprises ou administrations jouissent de conditions privilégiées : assurances, salaires confortables, droit au regroupement familial, logement de fonction, accès facilité aux prêts bancaires, scolarisation publique des enfants, entre autres. Ces bénéfices décroissent à mesure que les positions au sein des hiérarchies professionnelles s'abaissent. La mixité de genre diminue également à mesure que les postes sont moins qualifiés. 
11 En partie déterminées par les capitaux sociaux dont disposent les immigrés à leur arrivée aux Émirats, les différences de conditions professionnelles se répercutent sur les lieux de vie. Les cadres peuvent vivre en famille ou seuls dans des appartements individuels payés par leurs employeurs. Ils occupent les quartiers cossus des cités émiriennes, où se fréquentent des immigrés aux revenus similaires, quoique de nationalités variées. Les employés moins privilégiés, exerçant dans le secteur privé des professions de service ou manuelles, sont contraints à la promiscuité. Plutôt que d'être logés par leurs employeurs dans les dortoirs des très contrôlées habitations ouvrières périphériques, beaucoup usent de leurs aides au logement pour acquitter en colocation les loyers onéreux de leurs cités d'accueil. Ils cohabitent ainsi entre colocataires de même sexe, et souvent de même nationalité ou "aire culturelle", à raison de 5 à 6 individus par chambre.

\section{Immigration et contrôle des espaces urbains}

Outre l'habitat, les lieux de la ville témoignent de l'emprise de la discipline migratoire, à la fois imposée par l'administration émirienne et reproduite par les habitants, citoyens et immigrés confondus. Les inégalités entre arabes et asiatiques, entre cols blancs et cols bleus, influencent aussi les interactions extra-professionnelles. L'accès aux espaces de loisirs est contrôlé soit directement par l'administration, soit par des services privés de sécurité, soit par sélection pécuniaire ou communautaire du public. Le contrôle policier des espaces urbains s'exerce surtout à l'encontre des immigrés pauvres, identifiés par leur couleur de peau (ce dont pâtissent les travailleurs du souscontinent indien) et leurs tenues (ce qui frappe autant les immigrés asiatiques qu'arabes) (Bristol-Rhys, 2012). Rares sont les espaces ouverts à tous indistinctement.

Le filtre pécuniaire leur est également défavorable : aux Émirats, presque tout se paie, y compris l'entrée au parc et à la plage. Les malls et les bars des hôtels sont chers et les entreprises de gardiennage y assurent une sélection rigoureuse à l'entrée, selon des critères similaires à ceux pratiqués, dans la rue, par les autorités. Enfin, nombre de petits restaurants et cafés, les seuls abordables pour les immigrés désargentés, s'adressent explicitement à une clientèle communautaire, en vertu de la langue pratiquée par le personnel, de la teneur très spécifique des menus et de l'homogénéité culturelle des habitués. A Abu Dhabi, seule la corniche piétonne et ombragée est ouverte à tous, sans distinction de nationalités, d'habits, de couleurs de peau, de genres, d'âges ou de revenus - même si les interactions qui s'y déroulent sont en partie irriguées par ces rapports sociaux, intériorisés par les passants (Assaf, 2013). Les ordres structurel et interactionnel se prolongent.

Identifications de genre et de génération importent également dans les rapports des habitants aux espaces de la capitale émirienne. Les hommes seuls, considérés comme célibataires, ont ainsi l'interdiction de fréquenter les mêmes plages que les «familles ", ce dont s'assurent les sociétés de gardiennage précitées. Les inégalités par origines nationales s'imbriquent alors aux inégalités de classes - seuls les travailleurs qualifiés et aisés peuvent en effet obtenir le droit au regroupement familial - pour renforcer le filtre du public de la plage. Les jeunes sont en outre plus pénalisés que leurs aînés, car d'emblée réputés célibataires. Sur le chapitre du genre, le gouvernement supplée le contrôle semi-privatisé des espaces par la pénétration administrative de l'intimité des 
gouvernés, en décourageant les mariages mixtes et pénalisant la drague, par exemple (Hasso, 2011).

\section{Galérer, progresser, profiter : trois trajectoires singulières à Abu Dhabi}

Les contrôles du marché du travail et des espaces urbains modèlent les subjectivités de tous les résidents des Émirats arabes unis. Les immigrés égyptiens rencontrés n'échappent pas à la règle. Les trois portraits qui suivent reflètent les assujettissements et les socialisations inédites auxquelles expose personnellement et collectivement la condition d'immigré, tout en faisant ressortir l'importance des dispositions sociales pré-migratoires.

\section{Adam, le chômeur en quête de contrat}

16 Adam est un ami rencontré en 2003 au Caire, alors qu'il étudiait la comptabilité dans un institut technique public. Le jeune homme réside avec son père, sa mère et deux de ses frères dans l'appartement familial d'un quartier populaire de Giza. A l'issue de ses études, Adam a travaillé dans diverses entreprises privées au Caire. Lorsque nous nous retrouvons à Abu Dhabi, en décembre 2012, il cherche depuis un mois un contrat de travail lui permettant d'obtenir un permis de séjour. Fiancé à une jeune fille d'une famille aisée, Adam est parti pour accumuler la dot exigée par son futur beau-père. Il relate sa lassitude des contrats à 1500 livres par mois au Caire (165 euros), largement insuffisants pour économiser les 200000 livres (22000 euros) voués à l'achat d'un appartement, de la dot en or, des meubles et au financement de la fête de mariage. Trouver un contrat, Adam estime encore le devoir à son père, lequel a eu un accident cardiovasculaire lorsque son fils a démissionné de son emploi de comptable au Caire, quelques mois auparavant, en raison d'un différend avec son employeur. Sa famille lui a en outre prêté l'argent nécessaire au paiement du visa de tourisme et du billet d'avion.

17 Adam souligne l'insécabilité des dimensions économiques et genrées de l'expérience migratoire. C'est pour devenir un homme marié et indépendant qu'il a dû partir. Mais c'est aussi pour devenir un homme viril. En décembre 2012, à 28 ans, Adam est encore vierge. "Le corps a des exigences, c'est difficile d'attendre » soupire-t-il, comparant avec désespoir la relative liberté des relations amoureuses à Marseille (où je vis) à la nécessité d'en passer par le mariage - et les frais afférents - au Caire. Bien qu'enthousiaste devant la diversité des nationalités, des corps et des atours féminins à Abu Dhabi, Adam s'interdit la drague. Il craint de se faire expulser s'il s'adonnait à cette performance de virilité si commune au Caire. Pour autant, le jeune homme se félicite du strict encadrement légal des relations de séduction aux Émirats, regrettant qu'il n'en aille pas de même en Égypte, ce dont sa fiancée pâtit au quotidien.

$18 \mathrm{Au}$ cours des semaines que nous passons ensemble, Adam compare souvent sa difficulté à décrocher un contrat à la situation des membres de sa famille, émigrés à Abu Dhabi avant lui. L'une de ses tantes paternelles a quitté le Caire, vingt-cinq ans auparavant, pour travailler comme professeure de dessin dans le secteur public émirien ; elle a pu faire venir son mari et l'une de ses sœurs. Elle est aujourd'hui aisée et a investi dans d'onéreux cursus pour ses trois enfants, ainsi que dans un appartement dans un quartier chic du Caire. La seconde tante d'Adam travaille comme comptable au 
ministère des finances et mène aussi une existence confortable. Quant au frère aîné d'Adam, il est arrivé en 2006 à Abu Dhabi et a trouvé à s'employer comme comptable au ministère des finances, après divers contrats privés peu rémunérés. Jeune marié, il exerce deux emplois pour subvenir aux frais du ménage, au ministère le matin et dans une entreprise privée l'après-midi.

Les déboires d'Adam renvoient au caractère utilitaire du dispositif migratoire émirien. Aucune de ses démarches auprès de connaissances immigrées, de réponses à des annonces et de candidatures spontanées n'a abouti. La seule offre qui lui ait été faite, comme réceptionniste dans une agence de voyage, était sous-payée : 2500 dirhams par mois (520 euros) pour travailler le matin et de nuit, sans prime au logement ni prise en charge des frais de permis de séjour (3 500 dirhams soit 730 euros). Adam a donc décliné. Le comptable lie ses difficultés à la fois à sa piètre maîtrise de l'anglais et au fait que les autorités émiriennes profitent de la révolution entamée en janvier 2011 pour accroître la pression salariale sur les immigrés égyptiens, sous couvert de contention de la contestation politique.

En l'absence d'emploi, les loisirs d'Adam sont limités. Le jeune homme s'émerveille du confort et de l'organisation urbaine d'Abu Dhabi, des voitures rutilantes, des malls luxueux, des plages contrôlées, sans qu'il ne puisse en jouir en raison de sa précarité statutaire, économique et de son célibat. Adam passe ses soirées dans un café égyptien proche du domicile de son frère, dans un quartier central peuplé d'immigrés arabes. Au café, les clients, tous des hommes égyptiens, viennent fumer des narguilés et boire du thé noir, tout en discutant des dernières manifestations en Égypte. Hors de ces sorties, Adam passe ses journées sur Facebook et Skype à conter ses démarches infructueuses à sa famille et ses amis au Caire, sans prendre position vis-à-vis des évènements égyptiens par crainte de la censure politique émirienne. Il va aussi prier à la mosquée proche du domicile fraternel.

21 A la fin de mon séjour, Adam se lamente à propos de cette routine tissée de frustration, dont son corps se fait le miroir : «J'en ai assez de ne pas trouver de travail, de toujours solliciter les gens... Je ne veux pas d'un travail de merde, non plus. Le $1^{\text {er }}$ janvier, c'est décidé, je rentre en Égypte, même revêtu des habits de l'échec. Je ne veux plus rester ainsi, à ne rien faire, même mon corps a changé, je prends du ventre! Quand je ne paresse pas à l'appart, je vais à la mosquée, ça ce sont mes activités!» Adam finit par abdiquer ses rêves de confort et de richesse à Abu Dhabi pour revenir au Caire. En juin 2013, il parvient à se marier après avoir décroché un emploi dans sa branche. Les difficultés économiques accrues depuis la révolution, et la pugnacité amoureuse de sa fiancée, ont permis à Adam de convaincre sa belle-famille de renoncer à ses hautes ambitions matérielles. La pauvreté au pays plus que la fortune à l'étranger lui a permis de réaliser ses rêves matrimoniaux, tout en abdiquant ceux de fortune.

\section{Nasir, le révolté en quête de reconnaissance}

Je rencontre Nasir peu après mon arrivée, début décembre 2012, au bar d'un grand hôtel d'Abu Dhabi, en compagnie de jeunes hommes et femmes de diverses nationalités. Nasir explique dans un anglais parfait qu'il a grandi à Abu-Dhabi de ses dix à ses seize ans, puis est retourné étudier à Alexandrie, sa cité d'origine, pour revenir à Abu Dhabi en 2006. Il travaille comme responsable des crédits dans une grande banque française et vit avec son père, sa mère et sa sœur aînée dans un appartement en centre-ville. Lors 
de nos rencontres successives dans les bars de la ville, Nasir conte l'histoire migratoire de sa famille, qui commence avec son père, un mécanicien émigré à Abu Dhabi en 1979.

Spécialiste en poids lourds, le père de Nasir trouve du travail dans une entreprise privée. Il y exerce jusqu'en 1984, avant de revenir à Alexandrie avec en poche de quoi ouvrir un atelier et subvenir aux besoins de ses deux filles et de son fils. Nasir grandit dans le quartier pauvre alexandrin d'où sa famille est originaire. En 1993, son père repart à $A b u$ Dhabi, son petit atelier étant menacé de ruine par la prévarication bureaucratique égyptienne. Réemployé par son ancienne entreprise émirienne, le mécanicien trouve en 1994 un emploi dans le secteur public, et fait alors venir femme et enfants. Son épouse travaille pendant quelques années dans une crèche privée, à destination des enfants d'immigrés arabes non pris en charge par les écoles publiques.

L'adolescence émirienne de Nasir se déroule entre le logement de fonction qu'occupe sa famille, l'école publique et les loisirs amicaux plus ou moins interlopes. Ses voisins de palier sont Marocains, Indiens, Philippins, Palestiniens, si bien que Nasir apprend rapidement l'anglais et les autres dialectes arabes en compagnie de leurs enfants. Le quotidien du jeune garçon change drastiquement : «C'était de très belles années pour moi, le contraire de ma vie en Egypte! A Alex, j'avais l'habitude de jouer dans la rue avec les gamins du quartier, on s'insultait, on se tapait, on portait des pantalons troués, etc. Ici, il y avait de la civilité, de l'ordre ", se souvient-il.

Le contraste lui vaut le stigmate des élèves égyptiens rencontrés au collège et au lycée, qui méprisent l'origine sociale de Nasir. "Eux, ils parlaient de Play station, ce genre de trucs, quand moi, bien évidemment, avec un père mécano, je ne savais pas ce que c'était. Je ne venais pas de la même classe sociale qu'eux... En plus, j'étais débrouillard et je parlais avec toutes les autres nationalités, alors qu'eux, ils restaient entre eux, entre Égyptiens. Eux, ils étaient beaucoup plus riches que nous ». Nasir développe des amitiés durables avec Taha, un jeune Soudanais de famille aisée, " mais pas snob », avec ses voisins, ainsi qu'avec les enfants des amis égyptiens et palestiniens de sa mère. Les Émiriens en revanche ne lui sont pas accessibles, étant plus âgés et encore plus arrogants que les «fils de médecins, d'ingénieurs ou de professeurs " égyptiens. La césure entre citoyens et immigrés est d'autant plus vive qu'en 1998, au collège, une expérience officielle oblige les élèves émiriens à aller en cours le matin et les élèves étrangers l'après-midi.

Les loisirs adolescents de Nasir lui ont laissé de doux souvenirs. Il délaisse vite la Play station et les vidéos pornographiques pour les promenades dans les supermarchés et au Burger King, " pour regarder les filles », ainsi que dans une arcade de jeux vidéo, Electra, qui a si bien marqué une génération d'habitants d'Abu Dhabi qu'elle a informellement donné son nom à l'une des artères de la ville. Lors de ces pérégrinations, Nasir rencontre, à quinze ans, une Seychelloise du nom de Lala, avec laquelle il s'initie aux French kisses dans les maisons traditionnelles abandonnées de la côte, aujourd'hui remplacées par d'imposants buildings. En compagnie de Lala, des camarades philippines de la jeune fille et de Taha, Nasir passe ses soirées entre barbecues à la plage et flâneries dans les malls qui poussent en ville comme des champignons. Le jeune homme achève alors de globaliser ses sociabilités, écoutant les derniers tubes américains de MTV, dégustant des hamburgers entre autres spécialités exotiques, se familiarisant avec les cartes de Saint Valentin et se perfectionnant en langue anglaise.

Nasir vit les affres de la rupture amoureuse peu avant de retourner à Alexandrie pour y commencer ses études à la faculté publique de commerce international, en 2001. Rentré 
seul, il redécouvre à dix-sept ans son quartier pauvre d'origine. Le retour est " comme une révolution " pour le jeune homme, en raison du cadre de vie où " tout est sale, rien ne marche "... En outre, son grand-père, son oncle et ses tantes alexandrins le considèrent comme un enfant gâté car ses parents le financent à distance (à raison de 300 livres - 33 euros - par mois). «L'Égypte, pour moi, c'était une vie de fer après une vie de miel », résume-t-il. Et ce d'autant que la difficulté du retour est accrue par l'incompréhension de ses camarades à l'université, devant le contraste entre la profession dévalorisée de son père, son quartier populaire de résidence, et ses manières "globalisées ». Nasir décide en outre de ne jamais entretenir de relation amoureuse avec des Égyptiennes, tant les préoccupations matérialistes de ses camarades, pour lesquelles le mariage est une clef d'ascension sociale sous couvert de prodigalité masculine, lui semblent aliénantes pour les femmes comme pour les hommes.

Pris dans les tenailles de la lutte de classes égyptienne et de ses répercussions genrées, sans pouvoir s'identifier ni à la bourgeoisie estudiantine, ni à sa famille alexandrine, Nasir dit avoir si bien souffert qu'il a "retourné [son] sentiment d'injustice contre Dieu ", devenant «à peu près athée ». Sa "révolution» existentielle s'est encore matérialisée par une consommation frénétique de haschich et l'écoute de heavy metal avec un groupe d'amis, comme lui marginaux à l'université. L'un d'entre eux composait des textes très hostiles à Moubarak. "Nous étions tous convaincus que le système devait changer, qu'il fallait des révolutions. Ben, finalement on l'a eue ", grimace Nasir. A la révolution, le jeune homme a cependant préféré l'émigration.

En 2006, à l'issue de sa licence, il revient à Abu Dhabi, alors que son père vient d'être mis en retraite de son emploi public. Les immigrés perdant leur droit au séjour sans contrat de travail, le père de Nasir s'engage dans le privé pour un salaire bien moindre, si bien que son fils cherche un emploi afin de soutenir le ménage. Arrivé avec un visa de tourisme, Nasir décroche un poste dans la banque française où il travaille toujours, grâce à l'entregent maternel et à sa maîtrise de l'anglais. D'un salaire de 4000 dirhams à l'essai (830 euros), il passe progressivement à son revenu actuel de 16000 dirhams (3 330 euros), qui lui servent à payer la moitié du loyer de 6000 dirhams (1250 euros), ainsi que ses études en gestion d'entreprise dans une université écossaise privée. Nasir se dit fier d'être indépendant et responsable de sa famille.

Dans la banque qui l'emploie, les hiérarchies par nationalités sont sensibles en termes de salaires - les cadres indiens gagnent ainsi moins que les Arabes, sans qu'elles ne se répercutent sur les interactions entre collègues. Outre ces derniers, Nasir fréquente des jeunes gens de toutes nationalités rencontrés par coach surfing, comme sa copine actuelle, une professeure néo-zélandaise. Quant à son meilleur ami, Égyptien comme lui, il est lié au groupe de heavy métal alexandrin auquel Nasir a participé durant ses études. Les deux compères ont en commun un intérêt appuyé pour la politique égyptienne, ainsi qu'un penchant pour les soirées éthyliques dans les bars d'Abu Dhabi. Si depuis son retour, en 2006, Nasir estime que son but est de profiter de la vie, ses journées sont éreintantes, partagées entre travail, études, famille, amours et sorties nocturnes.

31 En outre, de la «double absence » ressentie au gré de son périple égypto-émirien, le jeune banquier a tiré un répertoire critique tant sur sa société et ville d'origine que sur celles où il séjourne. Enthousiasmé par la déposition de Moubarak, il regrette de ne pas avoir été sur place pour y participer, et dénonce tant l'ignorance politique qui a mené, selon lui, aux victoires électorales des Frères musulmans, que l'incapacité du 
gouvernement Morsi à lutter contre les inégalités sociales égyptiennes. Quant aux Émirats, Nasir ne les envisage que comme une transition vers un autre lieu de vie, loin $\mathrm{du}$ contrôle politique du régime des cheikhs et de la superficialité de l'ethos consumériste dominant. Interrogé sur les contradictions apparentes entre ses convictions et son emploi dans la finance internationale, Nasir explique qu'il envisage le second également comme une transition, en attendant de trouver un emploi qui lui siérait davantage à l'issue de ses futures études en Ecosse.

\section{Galal, le yuppie en quête de plaisirs}

Le contact de Galal m'a été transmis par une connaissance émigrée en France. Galal est originaire du quartier bourgeois d'Héliopolis, au Caire. Il est issu d'une grande famille de propriétaires terriens touchés par la réforme agraire nassérienne, mais parvenus à se ménager, après 1961, de confortables sinécures bureaucratiques. Fils de magistrat, Galal a dès avant son départ pour le Golfe investit l'héritage paternel dans un appartement de la très cossue communauté fermée de Rihâb («La spacieuse »), à l'Est du Caire. Âgé de trente-cinq ans, il possède un diplôme en audit financier de l'université publique d'Ain Chams, complété par un cursus de deux ans dans le Minnesota. Galal a cherché à s'employer aux Émirats arabes unis pour faire carrière. Il s'est aussi plié ce faisant aux vœux de sa mère, qui ne souhaitait pas voir partir son benjamin trop loin d'Égypte. Au volant d'une rutilante Mercedes décapotable, vêtu avec raffinement (bermuda et chemise en lin, chaussures, montre et lunettes de prix), il m'emmène bruncher dans un restaurant libanais réputé pour y conter son expérience émirienne.

Arrivé à Abu Dhabi en 2005, Galal travaille aujourd'hui pour une grande entreprise privée liée au ministère des finances émirien. Professionnellement, le jeune cadre se dit très satisfait : il gagne 48000 dirhams par mois (10000 euros), un salaire mirobolant qui inclut des primes au logement et à l'ameublement. Galal dispose de toutes les assurances auxquelles peut prétendre un immigré qualifié : accident, santé, billets d'avion, prêts bancaires à taux zéro. La kafâla est assurée collectivement sur l'entreprise. Galal travaille avec des collègues de toutes origines, mais a essentiellement sympathisé avec les Égyptiens, les Émiriens restant distants et les autres nationalités suscitant sa suspicion. Surtout, les collègues égyptiens de Galal sont d'anciens camarades de faculté : la spécialisation en audit financier étant très sélective au Caire, les élèves s'y connaissent tous. Célibataire, le jeune homme vit seul dans un confortable appartement meublé, dont l'entretien est assuré par une bonne asiatique, dans un building récent du centre-ville d'Abu Dhabi.

Hors du travail et de son chez-lui, Galal avait pour habitude de passer ses soirées dans les bars de Dubaï et d'Abu Dhabi en compagnie d'amis égyptiens également aisés et célibataires. Depuis le mariage de l'un et le retour de l'autre en Égypte, Galal a dû mettre un terme relatif à ces sorties. Grâce aux réseaux sociaux anglophones de type coach surfing et meets me, il a surmonté sa solitude et rencontré des "expats" de diverses nationalités, avec lesquels il part faire des safaris dans le désert ou jouer au paint-ball et au bowling dans les malls. Par ailleurs, lors de notre rencontre, Galal revient tout juste d'un séjour au Népal en compagnie de collègues égyptiens, passé à faire du rafting, du saut à l'élastique et de la randonnée, en évitant soigneusement les spécialités 
culinaires locales. La Thaïlande avait précédé le séjour népalais, premiers pas vers d'autres voyages en projet.

Toujours en compagnie d'amis égyptiens, Galal a fréquenté quelquefois les « discos » émiriennes, hauts lieux de prostitution. Comme les autres professions, l'exercice du plus vieux métier du monde est structuré par les inégalités entre travailleurs de différentes nationalités ou aires culturelles. Les travailleuses du sexe arabes et esteuropéennes sont les plus recherchées par les clients émiriens et immigrés riches, tandis que celles d'Asie centrale, des Philippines, de Chine ou d'Éthiopie monnaient leurs corps à destination d'une clientèle moins fortunée. Galal n'a plus besoin de recourir aux relations sexuelles tarifées, cependant, car les relations sociales cosmopolites tissées via les réseaux sociaux en ligne lui permettent de rencontrer des jeunes femmes de divers coins du globe, avec lesquelles il lui arrive de nouer des relations amoureuses récréatives ou durables. Les femmes d'Europe de l'Est ont sa préférence, «car elles ont le sens du respect de l'homme : elles font attention à lui, lui préparent à manger etc. Un peu comme les Arabes, tu vois ", s'enthousiasme-t-il.

Questionné en ce sens, Galal répond ne jamais fréquenter d'Asiatiques, ce qu'il ne faut pas interpréter comme du racisme de sa part, affirme-t-il, mais plutôt comme une affaire de classes. Ainsi Galal se refuse-t-il aussi à fréquenter des Égyptiens d'un autre milieu social. Il explique: "À la rigueur, je préfère sortir avec des Pakistanais ou d'autres nationalités qui sont d'un niveau social inférieur, plutôt qu'avec des Égyptiens plus pauvres, pour me prémunir du mauvais œil... L'Égyptien, il ne pourra pas s'empêcher de comparer entre ta situation et la sienne, il sera jaloux. Les Asiatiques, non. Ils sont habitués aux différences de niveaux de vie ». De toutes façons, hormis ses amis de facultés égyptiens, l'ensemble des relations sociales de Galal lui paraissent vouées au temporaire. En effet, "Les gens vont et viennent, ici, au gré de leurs contrats ».

37 C'est là le seul bémol de sa vie émirienne : bien qu'il apprécie le confort des Émirats arabes unis, Galal regrette qu'il demeure difficile de s'y projeter à long terme. Pour autant, il ne fait pas montre d'un engagement vif envers l'Égypte - à l'exception de ses visites très régulières à sa mère. La politique ne l'intéresse guère, ainsi, si ce n'est qu'il n'aime pas les Frères musulmans. De toute façon, conclut Galal, la majorité des habitants d'Abu Dhabi ne parlent pas politique, par crainte de la surveillance policière, et aussi parce qu'ils sont là pour le travail. Lui-même ne fait pas exception !

\section{Le changement social au prisme migratoire : une mosaïque tout en nuances}

La discipline anthropologique s'est attachée aux arts de résister des "subalternes", dont ceux des migrants. Ce faisant, elle a parfois délaissé la subtilité des rapports sociaux que manifestent les phénomènes migratoires, lesquels ne se résument guère à l'opposition entre dominants (la bourgeoisie de l'État de départ, l'administration et le patronat de l'État d'arrivée) et dominés (les émigrés devenus immigrés). «Le pouvoir est essentiellement ambigu ", rappelait Georges Balandier (1978, p. 204). En ce sens, les migrants sont toujours relativement dominés et relativement dominants : la mobilité est une matrice à rapports sociaux complexes, qui ne se réduisent ni à un 
assujettissement univoque, ni en une émancipation subreptice des rapports de pouvoir au pays comme en exil. Les relations à la ville manifestent et structurent ces rapports.

\section{N'est pas global qui veut : de l'importance des dispositions}

39 Les trois trajectoires exposées reflètent l'importance des différences entre émigrés, lesquelles s'altèrent, se maintiennent, voire s'exacerbent à Abu Dhabi. Pour Galal, l'émigration permet d'entrer dans les cercles des " cadres circulants internationaux ", lesquels reproduisent d'une ville et d'un pays à l'autre des styles de vie similaires - des appartements aux vêtements, des goûts culinaires aux loisirs (Tarrius, 1992, p. 130). La double expérience émirienne de Nasir le socialise à un style de vie consumériste globalisé, devenant l'occasion d'une ascension sociale autrement improbable. Son récit rend également compte de l'importance des contextes historiques sur les politiques et les trajectoires migratoires : les conditions de vie en Égypte et d'emploi aux Émirats arabes unis ont évolué de 1979 à 2012, vers davantage de précarité des travailleurs. Adam témoigne de la traduction subjective de ces évolutions : le jeune comptable marque en son âme et sa chair les difficultés à devenir un homme complet, à savoir actif, prodigue et viril, entre deux rives.

Les rapports de classes se renforcent comme ils se brouillent en immigration: la mobilité alimente les luttes de classement entre groupes sociaux (Adelkhah et Bayart, 2007). Adam reste cantonné à sa condition de diplômé prolétaroïde, évincé des délices consuméristes émiriens. Ses rêves cosmopolites se heurtent à la médiocrité de sa condition socioprofessionnelle et à sa nationalité tenue en suspicion politique. Égyptien, mal diplômé et pauvre, Adam cumule les attributs des «classes potentiellement dangereuses ", du point de vue des autorités émiriennes. De son côté, Galal conforte sa condition bourgeoise héritée, en l'adaptant aux exigences globalisées de son temps. Nasir vit les affres du déchirement entre deux classes, que symbolisent ses allers-retours entre Alexandrie et Abu Dhabi. Ce déchirement irrigue un répertoire critique des inégalités sociales égyptiennes et émiriennes. La distanciation sociale et géographique le porte à « relativiser sa condition originelle » (Sayad 1999, p. 137), mais aussi les fondements politiques des hiérarchies auxquelles il est exposé.

41 A l'instar de la classe, les rapports de genre sont marqués par les ruptures et socialisations que suscite l'expérience migratoire (Fouché et Weber, 2006). Le plus pauvre comme le plus riche des trois jeunes hommes se conforment au modèle patriarcal égyptien, mais ils le retouchent. Parti pour devenir un homme idéal, Adam finit par dépouiller le mariage de ses exigences pécuniaires, relativisant d'autant l'importance de la prodigalité masculine. Galal recourt à la prostitution et aux idylles avec des étrangères, pratiques qui siéent mal à la moralité endogame de l'homme égyptien modèle, pour pallier sa solitude sexuelle et affective. Il n'altère guère l'idéal domestique inégalitaire, néanmoins, mais le " globalise » en l'adaptant à des relations exogames. Quant à Nasir, les contrastes vécus entre son adolescence émirienne romantique et cosmopolite, doublés de la consubstantialité des inégalités par classe et par genre à Alexandrie, l'incitent à la critique du modèle matrimonial égyptien. A Abu Dhabi, cependant, il endosse auprès de sa famille le rôle masculin correspondant au modèle conspué - responsable, travailleur et prodigue. 


\section{Les espaces, supports et enjeux des luttes sociales}

42 Les trajectoires d'Adam, Nasir et Galal indiquent toutes trois la densité des expériences individuelles, liant politiques d'État, rapports sociaux inégalitaires, dispositions héritées et nouvelles socialisations d'une rive à l'autre. Les trois interlocuteurs jouent avec les échelles de pouvoir auxquelles les exposent leurs expériences migratoires : ni parfaitement rebelles à des dominations en miroir, ni purs victimes de ces dominations, ils les adaptent à leurs espaces quotidiens, en fonction de leurs socialisations ante et post-migratoires, ainsi qu'en regard des économies morales dans lesquels leurs trajectoires sont emprises (Fassin, 2012). D’Égypte aux Émirats, les expériences singulières travaillent et sont travaillées par les rapports de production et de redistribution (générateurs d'inégalités par classes, générations et nationalités), ainsi que par les systèmes de valeurs auxquels ces rapports s'adossent (ethos consumériste globalisé, représentations racistes, modèles genrés).

43 L'immigration égyptienne dans les Émirats est diverse : les trois expériences présentées en attestent par leurs contrastes. Il est dès lors délicat d'identifier les contours d'une économie morale dominée aux prises avec une économie morale dominante (émirienne, capitaliste, rentière, discriminatoire). Le concept ne définit pas les termes d'une lutte générique (immigrés égyptiens versus dispositif migratoire émirien), qui n'est point vécue comme telle par tous (et surtout pas par Galal), que pour souligner l'importance des dimensions symboliques des rapports de pouvoir, et leur insécabilité des dimensions matérielles (Halbwachs, 1975 ; Thompson, 1971, p. 78). La diversité des trois trajectoires ici comparées interroge également la pertinence du prisme communautaire dans le balisage du terrain d'enquête à Abu Dhabi. La nationalité est certes une catégorie primordiale de la stratégie migratoire émirienne du « diviser pour mieux régner ". Elle ne subsume cependant pas les autres identifications des enquêtés, lesquelles s'actualisent dans des contextes interactionnels et spatiaux déterminés.

44 Suivre les enquêtés du travail au mall, du café à la " disco ", de la plage à l'écran, permet d'observer les marges de jeu qu'ils investissent entre ordre structurel et interactionnel. Si la banque où exerce Nasir est imprégnée de hiérarchies par nationalités, en revanche, ses usages des malls comme lieux de drague cosmopolite et des maisons abandonnées comme site de rencontres amoureuses contournent les frontières communautaires, les dimensions morales et discriminatoires de l'ordre urbain. Pour Adam, au contraire, l'impossibilité d'accéder aux espaces de loisirs convoités le renvoie à l'échec de sa quête de réussite. Quant à Galal, des bowlings aux safaris dans le désert, de son confortable appartement aux discothèques, tous les espaces lui sont ouverts, à condition de déployer un savoir-être en contexte. Il s'agit de se plier aux règles tacites et explicites propres à chaque lieu : apprendre le mépris envers les serveurs indiens de ses restaurants favoris, savoir monnayer une passe en fonction de la nationalité de la prostituée, savoir séduire en anglais ses partenaires ukrainiennes de paint-ball...

Les capitaux économiques et culturels hérités, confortés par l'expérience migratoire, permettent à Galal de surmonter aisément les barrières sociales mises en espaces qui sont infranchissables à Adam et inspirent la révolte de Nasir. Ni simple décor, ni support rigide des rapports de pouvoir, les espaces tout à la fois les structurent et les altèrent (Agier, 1996). Les expériences des trois hommes présentés dans cet article se distinguent et évoluent en croisant leurs dispositions héritées, leurs socialisations nées de la migration et des règles interactionnelles adaptées au quotidien à Abu Dhabi. La 
consubstantialité de ces facteurs appelle à une pondération fine de leurs effets respectifs sur chaque trajectoire individuelle. Le singulier oblige à délaisser l'alternative analytiquement stérile entre domination et émancipation (Mauger, 2013), bien peu adaptée au phénomène migratoire et à ses protagonistes.

\section{BIBLIOGRAPHIE}

Adelkhah F., Bayart J-F., 2007. Introduction : pour une anthropologie politique du voyage. In Voyages du développement : émigration, commerce, exil, Paris, Karthala-CERI, p. 5-29.

Agier M., 1996. Les savoirs urbains de l'anthropologie. Enquête, nº 1, p. 35-58, en ligne sur http:// enquete.revues.org/document683.html, consulté le 28 septembre 2012.

Assaf L., 2013 (à paraître). La Corniche d'Abou Dhabi : espace public et intimités à ciel ouvert. Arabian Humanities, $\mathrm{n}^{\circ} 2$, numéro spécial «Villes et urbanisation dans la péninsule arabique contemporaine ».

Bartolomeo A., Fakhoury T., Perrin D., 2010. Egypte : profil migratoire. Florence, CARIM-IUE, en ligne sur http://www.carim.org/public/migrationprofiles/MP_Egypt_FR.pdf, consulté le 20 août 2013.

Bristol-Rhys J., 2012. Socio-spatial Boundaries in Abu Dhabi. In M. Kamrava et Z. Babar (dir.), Migrant Labour in the Persian Gulf, New York, Columbia University Press, p. 59-83.

Balandier G., 1978. L'anthropologie africaniste et la question du pouvoir. Cahiers internationaux de sociologie, vol. 65 , p. 197-211.

Dorlin E., 2009. Vers une épistémologie des résistances. In Sexe, race, classe : pour une épistémologie de la domination, Paris, PUF, p. 5-18.

Fassin D., 2012. Vers une théorie des économies morales. In J-S. Eideliman et D. Fassin (dir.), Économies morales contemporaines, Paris, La Découverte, p. 19-47.

Fouché N., Weber S., 2006. Introduction : construction des sexualités et migration. Migrances, $n^{\circ} 27$, p. $7-15$.

Gardner A., 2010. City of Strangers: Gulf Migration and the Indian Community in Bahrain. New York, Cornell University Press.

Halbwachs M., 1975. Esquisse d'une psychologie des classes sociales. Paris, Rivière.

Hasso F., 2011. Consuming Desires: Family Crisis and the State in the Middle-East. Stanford, Stanford University Press.

Kapiszewski A., 2006. Arab versus Asian Migrant Workers in the GCC Countries. United Nations Expert Meeting on International Migration and Development in the Arab Region, Beyrouth, 15-17 mai.

Lori N., 2010. Naturalization as 'National Security': The Political Management of Citizenship and the Growth of the Security Apparatus in the UAE since the 1990s. Gulf Research Meeting, Cambridge, 7-10 juillet. 
Mahdavi P., 2011. Gridlock: Labor, Migration and Human Trafficking in Dubai. Stanford, Stanford University Press.

Mauger G., 2013. La 'cause' des classes populaires. Présentation dans le cadre de la séance "Groupe dominés et changement social ». $5^{\text {ème }}$ Congrès de l'Association Française de Sociologie, Nantes, Université de Nantes, 2-5 septembre.

Müller-Mahn D., 2005. Transnational Spaces and Migrant Networks: A Case Study of Egyptians in Paris. Nord-Süd Aktuell, vol. 1, p. 20-33.

Passeron, J-C. Revel J., 2005. Penser par cas : raisonner à partir de singularités. In Penser par cas, Paris, EHESS, p. 9-44.

Saad R., 2007. Egyptian Workers in Paris: An Ethnographic Account. AUC Meeting on Migration and Refugee Movements in the Middle East and North Africa, Le Caire, 23-25 octobre, en ligne sur http://www.aucegypt.edu/GAPP/cmrs/Documents/ReemSaad.pdf, consulté le 25 août 2013.

Sayad A., 1999. La double absence : des illusions de l'émigré aux souffrances de l'immigré. Paris, Seuil.

Tarrius A., 1992. Les fourmis d'Europe : migrants riches, migrants pauvres et nouvelles villes internationales. Paris, L'Harmattan.

Thompson E., 1971. The Moral Economy of the English Crowd in the Eighteenth Century. Past and Present, vol. 50, $\mathrm{n}^{\circ} 1$, p. 76-136.

Vora N., 2013. Impossible Citizens: Dubai's Indian Diaspora. Durham, Duke University Press.

\section{NOTES}

1. Estimation délivrée par un ami égyptien exerçant comme cadre informatique dans une grande entreprise émirienne du nom de Watani (« ma patrie»). Parrainée par le cheikh al-Maktoum, gouverneur de Dubaï, Watani est notamment chargée de promouvoir «l'indigénisation» du marché du travail (tawtîn), c'est-à-dire le remplacement de la main-d'œuvre immigrée qualifiée par des travailleurs émiriens (entretien, Dubaï, décembre 2012).

2. D'après des données de la Banque mondiale, reprises par le CARIM (Di Bartolomeo, Fakhoury, Perrin, 2010).

3. Les noms sont fictifs.

\section{RÉSUMÉS}

L'immigration de travail égyptienne aux Émirats arabes unis suscite en Égypte des espoirs collectifs et individuels de changement social sans cesse recomposés. Mais les émigrés devenus immigrés ne sont pas des passeurs de changement univoques: les expériences migratoires s'inscrivent dans des histoires longues; elles se frottent à des dispositifs administratifs discriminatoires; elles marquent diversement les esprits et les corps des immigrés et les espaces qu'ils fréquentent. C'est ce que retrace cet article, à travers les trajectoires singulières de trois hommes égyptiens à Abu Dhabi. 
Egyptian labor migration to the United Arab Emirates gives rise to high and changing expectations in Egypt. Yet, migrants are not straightforward agents of social change: their experiences are historically framed; they put up with discriminatory administrative rules; migration brands minds and bodies, as well as the spaces where migrants live or which they cross. The article deals with this complexity through a compared analysis of three cases of male Egyptian immigrants in Abu Dhabi.

\section{INDEX}

Mots-clés : migration, Égypte, Émirats arabes unis, espace urbain, changement social

Keywords : migration, Egypt, United Arab Emirates, urban anthropology, social change

Thèmes : Sur le Champ - Sur le Terrain

\section{AUTEUR}

\section{LUCILE GRUNTZ}

Lucile Gruntz, lucilegruntz@yahoo.fr, est Docteure en anthropologie sociale, EHESS-Centre de recherches historiques, membre de l'ANR SYSREMO (Géographies de la mondialisation : émergence d'un système régional au Moyen-Orient, Rennes 2) et du programme ECOMIG (IRD, Marseille). Elle a publié récemment :

- Gruntz L., 2014 (à paraître en janvier). Poules aux œufs d'or, petits patrons ou pigeons ? Usages et mésusages de l'argent des émigrés de retour du Golfe au Caire. Autrepart, $n^{\circ} 68$, numéro thématique « L'argent des émigrés ».

- Gruntz L., 2012. La révolution du Golfe au Caire ? Migration de travail dans les monarchies pétrolières et changements sociopolitiques en Égypte. Migrations et Sociétés, vol. 24, n 143 , numéro thématique « Du Maroc à Bahreïn, des migrations en zones de turbulences ", p. 73-86. - Gruntz L., Cantini D., 2010. Des nouveaux riches aux jeunes martyrs : les évolutions de la migration de travail égyptienne au prisme de ses représentations médiatiques. In Iman Farag (dir.), Chroniques égyptiennes 2008, CEDEJ, Le Caire, p. 79-118. 